\title{
Inter-breath-hold Registration for the Production of High Resolution Cardiac MR Volumes
}

\author{
Nicholas M.I. Noble ${ }^{1,2}$, Redha Boubertakh ${ }^{1,2}$, Reza S. Razavi ${ }^{2}$, \\ and Derek L.G. Hill ${ }^{1}$ \\ ${ }^{1}$ Centre for Medical Image Computing, University College London, UK \\ $\{$ N.Noble, Derek.Hill\}@ucl.ac.uk \\ 2 Imaging Sciences, Guy's Hospital, Kings College London, UK \\ \{Redha.Boubertakh, Reza.Razavi\}akcl.ac.uk
}

\begin{abstract}
High resolution MRI images of the beating heart permit observation of detailed anatomical features and enable quantification of small changes in metrics of cardiac function. To obtain approximately isotropic sampling with an adequate spatial and temporal resolution, these images need to be acquired in multiple breath-holds. They are, therefore, often affected by through-plane discontinuities due to inconsistent breath-hold positions. This paper presents a method to correct for these discontinuities by performing breath-hold-by-breath-hold registration of high resolution 3D data to radial long axis images. The corrected images appear free of discontinuities, and it was found that they could be delineated more reproducibly than uncorrected images. This reduces the sample size required to detect systematic changes in blood pool volume by $57 \%$ at end systole and $78 \%$ at end diastole.
\end{abstract}

\section{Introduction}

High resolution dynamic 3D volumes of the beating heart are very desirable from both clinical and image processing points of view. Clinically, they permit observation of small anatomical features and enable quantification of small changes in metrics of cardiac function. In addition as we will show, intra-observer variability when delineating such images would appear to be greatly reduced when compared to the repeated delineation of traditional multi-slice cine functional images which have much lower through-plane resolution. From an image analysis perspective, volumetric imaging with isotropic resolution and a sinc point spread function in all directions is desirable because it improves the performance of multi-planar re-formatting and more sophisticated 3D analysis techniques. This should improve the performance of segmentation and model construction techniques, making automatic quantitative image analysis more reliable, and therefore lead to more clinically straightforward imaging protocols.

Currently, clinical MR images used for ventricular function assessment typically feature reconstructed voxel sizes of around $1.5 \times 1.5 \mathrm{~mm}$ in-plane and $8-10 \mathrm{~mm}$ through plane. Two or three slices of this type can be acquired in a single breath-hold, which leads to acquisition times of 4-6 breath-holds for coverage of the entire left ventricle in the short axis. Although these images demonstrate very good contrast, in the z-direction, the resolution is not sufficient to differentiate small features and the point spread function is non-ideal. In this paper, we acquire $3 \mathrm{D}$ volumes with reconstructed voxel sizes 
of $1.5 \times 1.5 \mathrm{~mm}$ in-plane and $1.5 \mathrm{~mm}$ through plane in chunks of 10 slices during $7-8$ breath-holds. During scanning the subject is instructed to hold their breath at the same position, but many patients find this very difficult in practice. Breath-hold inconsistencies result, most clearly visible as jagged structure boundaries in re-formatted long axis images. Translations of up to $23.5 \mathrm{~mm}$ and rotations of up to $8^{\circ}$ due to inconsistent breath-hold positions have been recorded in the literature [1].

Several approaches may be employed to remove through-plane discontinuities. Acquisition approaches include the use of respiratory navigators [2] which when placed through the diaphragm may be used to reject inconsistent breath-holds [3]. However this can greatly increase the scan time as the subject repeatedly attempts to hold their breath within a gating window of $2.5 \mathrm{~mm}$. Swingen et al. [4] corrected for in-plane translations by fitting a polynomial through the centroids of manually delineated in-slice endocardial contours. Each slice was then translated by the residual error following fitting. Lötjönen et al. [5] corrected for 3D translations by registering parallel long axis images and short axis images. Chandler et al. [6] corrected for 3D translations and rotations by registering short axis images to $3 \mathrm{D}$ volumes. However, these three techniques all seek to correct for breath-hold discontinuities in thick sliced images whose features are somewhat different to those of high resolution near-isotropic images. To date, only Moore et al. [7] have attempted to correct for breath-hold inconsistencies in images with high through-plane resolution. They performed successive rigid registrations between each slice and sagital and axial scout slices. This permitted translations in the foot-head and right-left directions to be corrected for. Their images however suffered from very poor SNR. To surmount this, they combined images from multiple subjects (via elastic registration and signal averaging) to form a high resolution atlas.

In this work, to correct for breath-hold discontinuities, 3D translations and rotations are recovered by registration of $2 \mathrm{D}$ long axis images acquired with radially oriented slices (figure 1) to high resolution 3D short axis multi-chunk images. Analysis is then performed to determine whether the described technique can be used to reduce the sample size required to detect systematic changes in blood pool volume. Such a reduction is potentially of great importance when performing clinical trials.

\section{Data}

Following plan scans and identification of the vertical and horizontal long axes, short axis ECG gated steady state free precession 3D volume images with SENSE factor 2 were obtained in 5 healthy male volunteers. Seventy to eighty slices were acquired in 7 or 8 chunks of 10 slices per end-expiration breath-hold. The field of view was adapted for each subject. Imaging parameters were: field of view $330-420 \mathrm{~mm}$, acquisition matrix $129 \times 160$ with $80 \%$ phase encode direction sampling in the in-plane direction, $50 \%$ phase encode direction sampling in the through-plane direction, reconstructed to $256 \times 256 \times 70-80$ giving a resolution of $1.30 \times 1.30 \times 1.57 \mathrm{~mm}-1.64 \times 1.64 \times 1.57 \mathrm{~mm}$, with 8 phases in the cardiac cycle, flip angle $45^{\circ}$, TE $1.5 \mathrm{~ms}$ and TR $3.08-3.54 \mathrm{~ms}$. An end diastolic ECG gated steady state free precession long axis image containing twelve 2D slices radially oriented about the long axis was then acquired during a single end-expiration breath-hold. The imaging parameters were; slice thickness $8 \mathrm{~mm}$, 
field of view $400-500 \mathrm{~mm}$, acquisition matrix $160 \times 192$ with $120 \%$ phase encode direction sampling, reconstructed to $256 \times 256$ giving a resolution of $1.56 \times 1.56 \times 8 \mathrm{~mm}-$ $1.94 \times 1.94 \times 8 \mathrm{~mm}$, flip angle $50^{\circ}$, TE $1.5 \mathrm{~ms}$ and TR $3.39-3.57 \mathrm{~ms}$. To enable subsequent variability assessment, this procedure was repeated twice during the same scan session for each volunteer.The images were acquired on a Philips Intera $1.5 \mathrm{~T}$ with master gradients, using a 5 element cardiac synergy coil and vector ECG.

\section{Methods}

Before registering the radial long axis and short axis multi-chunk images, transformations must be defined that relate any point in the short axis image to the corresponding point in the radial long axis image. Radial long axis images are stored by stacking radially adjacent images - figure 1 .

For each slice $(i)$, locations in voxel coordinates can be related to their position in world coordinates by the following matrix

$$
A_{R L(i)}=T_{i} R_{x i} R_{y i} R_{z i} O_{i}
$$

where $R_{x i}, R_{y i}$ and $R_{z i}$ are $4 \times 4$ matrices representing rotations about the respective $\mathrm{x}$, $\mathrm{y}$, and $\mathrm{z}$ axes, $T_{i}$ contains translations in the $\mathrm{x}, \mathrm{y}$ and $\mathrm{z}$ directions and $O_{i}$ is an orientation matrix with three possible values (corresponding to transverse, sagital and coronal slice orientations). The parameters that make up these matrices were automatically recorded during acquisition and subsequently extracted from the Philips proprietary PAR header file. Likewise, locations in voxel coordinates in the short axis image are related to their position in world coordinates by matrix $A_{S A}$ (composed as per equation 1 , but valid for the entire short axis image).

Pseudo radial long axis images were then created from the end diastolic multi-chunk images. For each ten slice chunk, a corresponding short axis 'chunk of interest image' was created. These images were the same as the short axis image, however all voxels that were not acquired during the breath-hold corresponding to that chunk were set to a padding value. The padding value was chosen so that it could not occur elsewhere in the image ( -1 in this case). Blank images with the same geometry as the radial long axis image were then created for each chunk of interest image - the pseudo images. For

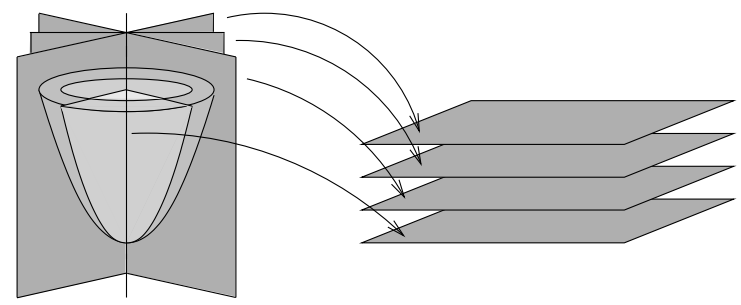

Fig. 1. Illustration of the orientation of radial long axis slice planes with respect to the left ventricle (left), and how they are stored in the image (right) 
each pseudo image, the voxel locations were transformed into world coordinates before being transformed into voxel coordinates in the short axis image.

$$
\operatorname{Pos}_{S A}=A_{R L(i)} A_{S A}^{-1} \operatorname{Pos}_{R L}
$$

where $\operatorname{Pos}_{R L}$ is a $4 \times 1$ matrix representing the voxel location in the radial long axis image and $\operatorname{Pos}_{S A}$ represents the corresponding voxel location in the short axis image. These locations were then interpolated using trilinear interpolation to provide the voxel intensity values for the pseudo radial long axis images. Figure 2 shows an example of several slices from pseudo radial long axis images.

To avoid the two stage interpolation associated with transforming the chunk of interest image prior to creating a pseudo radial long axis image, the registration transformation matrix was incorporated into the process of creating the pseudo images. Equation 2 hence becomes

$$
\operatorname{Pos}_{S A}=A_{R L(i)} T_{R E G} A_{S A}^{-1} \operatorname{Pos}_{R L}
$$

where $T_{R E G}$ is a $4 \times 4$ matrix which represents a six degree of freedom rigid body transformation.

The correlation coefficient similarity measure between the radial long axis image and each pseudo long axis image was then optimised as a function of the six degrees of freedom of $T_{R E G}$ using a simplex search method [8]. Voxels set to the padding value did not contribute to the similarity measure. A two stage optimisation approach was employed; firstly the $\mathrm{x}, \mathrm{y}$, and $\mathrm{z}$ translations alone were optimised, then all six parameters were optimised. The chunk of interest images were then transformed using trilinear interpolation according to their respective registration matrices. The corrected chunk of interest images were then combined into a single corrected image. Where multiple chunks overlapped, the mean voxel intensity was used. Following correction, small gaps may occur in locations where chunks did not overlap as shown in figure 2 The location of such gaps may be identified by searching for voxels which contain padding values in all of the corrected chunk of interest images. To fill these gaps, for each gap voxel, one dimensional linear interpolation was performed between the nearest non-gap voxels in the inferior and superior z-directions.

For each pair of radial long axis and short axis multi-chunk images, each chunk of the end diastolic multi-chunk image was registered to the radial long axis image. The registration matrices were then used to create corrected short axis image series. The end diastolic and end systolic blood pool volumes were then manually delineated in the original and corrected images by an expert observer using Analyze (Mayo Clinic, Rochester, MN, US).

The reproducibility of manually determined end diastolic and end systolic volume for the original and corrected images was investigated as per [9]. The mean and standard deviation difference between results and the mean and standard deviation percentage variability (defined as the absolute difference of two measurements divided by their mean) were assessed. The correlation coefficient between the first and second set of acquisitions was also calculated. Student's paired $t$-test was employed to identify any significant differences between the two sets of measurements. The sample sizes $(N)$ required to detect a systematic change $(\delta)$ of $1 \mathrm{ml}$ with a power $(P)$ of $90 \%$ and an $\alpha$ error of $5 \%$ were calculated using the following formula 


$$
N=f(\alpha, P) 2 \sigma^{2} / \delta^{2}
$$

where $\sigma$ is the standard deviation of the difference as described by Altman [10] and $f(\alpha, P)=10.5$ for $\alpha=0.05$ and $P=90$.

\section{Results}

Visually, all images registered well. Figure 2 shows example radial long axis views of the corrected images. Note specifically the areas around the right and left ventricular myocardium. The improvements in though-plane continuity can clearly be observed. The uncorrected images can show features that are not present in the corrected images and vice versa. This is due to rotations about the $\mathrm{z}$-axis being recovered during registration. The corrected images after filling contain residual intensity fluctuations resulting from the z-axis linear interpolation.

Table 1 shows the reproducibility data. It can be seen that for the corrected images the mean and standard deviation volume differences at both end diastole and end systole are reduced with respect to the uncorrected images. The mean and standard deviation percentage variability for both end diastolic and end systolic volumes of the corrected volumes were also less than for the uncorrected images. For both corrected and uncorrected images the correlation of both end diastolic and end systolic volume between the first and second set of images was very high (correlation coefficient $>0.98$ ). No significant differences between the first and second sets of images were observed $(p<0.05)$.

To detect a change of $1 \mathrm{ml}$ in end diastolic and end systolic volume in the uncorrected images requires 83 and 67 subjects respectively. To detect the same change in corrected

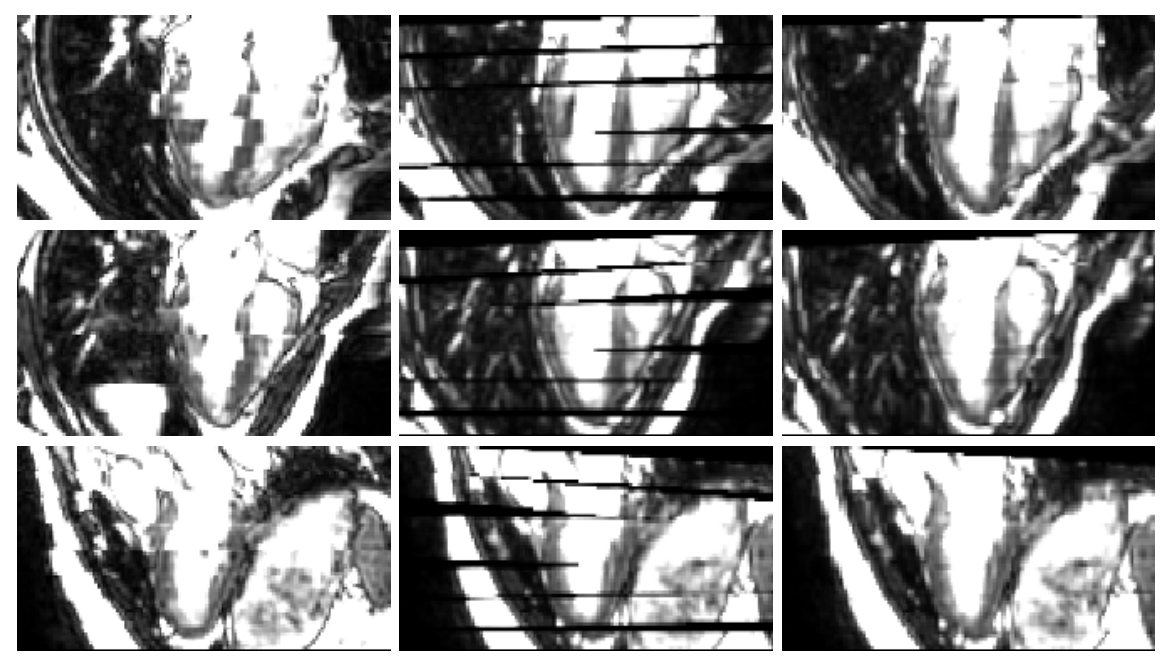

Fig. 2. Pseudo radial long axis images before correction (left), following correction (middle) and following correction and z-direction interpolation to fill any gaps (right) 
Table 1. Reproducibility data for uncorrected and corrected images

\begin{tabular}{lcccc}
\hline & \multicolumn{2}{c}{ Uncorrected } & \multicolumn{2}{c}{ Corrected } \\
& EDV $(\mathrm{ml})$ & ESV $(\mathrm{ml})$ & EDV $(\mathrm{ml})$ & ESV $(\mathrm{ml})$ \\
\hline Mean difference \pm SD & $3.07 \pm 1.99$ & $3.36 \pm 1.79$ & $1.17 \pm 0.92$ & $1.44 \pm 1.18$ \\
Correlation coefficient & 0.99 & 0.98 & 1.00 & 1.00 \\
$t$-test $p$ & $\mathrm{NS}$ & $\mathrm{NS}$ & $\mathrm{NS}$ & $\mathrm{NS}$ \\
$\%$ Variability $\pm \mathrm{SD}$ & $2.25 \pm 1.28$ & $6.94 \pm 3.94$ & $0.97 \pm 0.69$ & $3.55 \pm 2.56$ \\
\hline
\end{tabular}

$\mathrm{EDV}=$ End Diastolic Volume; ESV = End Systolic Volume; SD = Standard Deviation.

images requires 18 subjects for end diastolic volume and 29 subjects for end systolic volume. In percentage terms, these represent reductions in sample size of $78 \%$ and $57 \%$ respectively.

It is interesting to note that the standard deviations of differences in end diastolic and systolic volumes in both corrected and uncorrected images are much smaller than when delineating the thick slices exhibited by 2D multislice images - the current gold standard. Standard deviations of $4.3-4.7 \mathrm{ml}$ at end systole and $2.3-3.5 \mathrm{ml}$ at end diastole have been reported in the literature when manually delineating $10 \mathrm{~mm}$ thick slices [911]. To put these values in context, this would correspond to sample sizes of $388-464$ for end systolic volume and $165-257$ for end diastolic volume to detect a $1 \mathrm{ml}$ volume change with $P=90 \%$ and $\alpha=0.05$. This indicates that the reproducibility of end diastolic and end systolic volumes is much greater for high resolution 3D images.

\section{Discussion}

We have described a technique for generating high quality dynamic cardiac MR volumes from data acquired using multiple breath-holds. The approach corrects for inconsistent breath-hold positions by registration to a radial stack of long axis slices. We have demonstrated the qualitative benefit of this approach, and also used a power analysis to demonstrate that our technique can provide images that are more sensitive to within- and between-subject changes in cardiac function than the current gold standard technique of multi-slice cine cardiac MRI.

The radial long axis images have a slice thickness of $8 \mathrm{~mm}$, whereas the pseudo images are created by sampling high resolution images and have an effective thickness of around $1.5 \mathrm{~mm}$. Even at perfect alignment, the radial and pseudo images will appear slightly different because of the disparate slice thicknesses. A possible solution is to simulate thick slices by sampling the chunk of interest images at multiple points normal to the radial long axis slice plane. Weighted combination according to the slice select profile [12] would then produce a simulated thick sliced pseudo radial long axis image, which should better resemble the radial long axis image and may aid registration.

This work has assumed to good effect that misalignment due to inconsistent breathhold positions can be corrected for by using a rigid transformation model. However, the heart, chest wall and other organs move relative to one another during respiration. In addition, the shape of the heart alters during the respiratory cycle due to changes in the venous return. To further improve registration accuracy, it may be necessary to employ non-rigid registration. 
As was seen in figure 2 the corrected images contain residual intensity modulation in the areas of gaps in the data. These intensity modulations could be reduced by using more sophisticated through-slice interpolation (e.g. [13]), or alternatively using the inherent oversampling in each 3D chunk to fill the gaps. The latter approach would require working with the raw data, rather than the exported images, as this oversampled data is discarded prior to image exportation.

\section{Conclusion}

To conclude, a technique has been described for the correction of breath-hold discontinuities in high resolution short axis images via registration with radial long axis images. Visually, the results are very positive, providing a qualitative indication that the technique has effectively corrected for discontinuities resulting from inconsistent breathhold positions. Numerical analysis of delineations performed prior to and following correction has shown that correction substantially improves reproducibility. When these results are interpreted in terms of sample size, this improvement corresponds to a reduction of the sample size required to detect systematic changes of $1 \mathrm{ml}$ in blood pool volume of between $57 \%$ and $78 \%$.

\section{Acknowledgements}

This work was funded by the Engineering and Physical Sciences Research Council, grant GT/T11395/01. We are also grateful to Philips Medical Systems Nederland B.V. for research support.

\section{References}

1. K. McLeish, D.L.G. Hill, D. Atkinson, J.M. Blackall, and R. Razavi. A Study of the Motion and Deformation of the Heart Due to Respiration. IEEE Trans. Med. Imaging, 21(9):11421150, 2002.

2. Y. Wang, P.J. Rossman, J.P. Debbins, and R.L. Ehman. 3D Coronary MR-Angiography in Multiple Breath-Holds using a Respiratory Feedback Monitor. Magn. Reson. Med., 34(1):11-16, 1995.

3. R.S. Razavi, D.L.G. Hill, V. Muthurangu, M.E. Miquel, A.M. Taylor, S. Kozerke, and E.J. Baker. Three-Dimensional Magnetic Resonance Imaging of Congenital Cardiac Anomalies. Cardiol. Young, 13:461-465, 2003.

4. C. Swingen, R.T. Seethamraju, and M. Jerosch-Herold. An Approach to the ThreeDimensional Display of Left Ventricular Function and Viability using MRI. Int. J. Comput. Vision, 19:325-336, 2003.

5. J. Lötjönen, M. Pollari, S. Kivistö, and K. Lauerma. Correction of Movement Artifacts from 4D Cardiac Short- and Long-Axis MR Data. In C. Barillot, D.R. Haynor, and P. Hellier, editors, Medical Image Computing and Computer-Assisted Intervention-MICCAI 2004, pages 405-412, 2004.

6. A.G. Chandler, T. Netch, J.A. Schnabel, R. Razavi, D.L.G. Hill, and D.J. Hawkes. Correction of Misaligned Slices in Multi-Slice MR Cardiac Examinations by using Slice-to-Volume Registration. In D. Rueckert, J. Hajnal, and G.-Z. Yang, editors, Medical Image Understanding and Analysis - MIUA 2004, pages 33-36, 2004. 
7. J. Moore, M. Drangova, M. Wierzbicki, J. Barron, and T. Peters. A High Resolution Dynamic Heart Model Based on Averaged MRI Data. In R. Ellis and T. Peters, editors, Medical Image Computing and Computer-Assisted Intervention-MICCAI 2003, pages 549-556, 2003.

8. J.C. Lagarias, J.A. Reeds, M.H. Wright, and P.E. Wright. Convergence Properties of the Nelder-Mead Simplex Method in Low Dimensions. SIAM Journal of Optimization, 9(1):112-147, 1998.

9. N.G. Bellenger, L.C. Davies, J.M. Francis, and D.J. Pennell. Reduction in Sample Size for Studies of Remodeling in Heart Failure by the Use of Cardiovascular Magnetic Resonance. J. Cardio. Magn. Reson., 2(4):271-278, 2000.

10. D.G. Altman. Practical Statistics for Medical Research. Chapman and Hall, 1991.

11. F. Grothues, G.C. Smith, J.C.C. Moon, N.G. Bellenger, P. Collins, H.U. Klein, and D.J. Pennell. Comparison of Interstudy Reproducibility of Cardiovascular Magnetic Resonance with Two-Dimensional Echocardiography in Normal Subjects and in Patients with Heart Failure or Left Ventricular Hypertrophy. Am. J. Cardiol., 90(1):29-34, 2002.

12. A.G. Chandler. Improved Slice to Volume Registration using Information-Based Registration and Super-Sampling for Brain MR, Cardiac MR and Lung CT Applications. PhD thesis, Kings College London, 2005. In Press.

13. G.P. Penney, J.A. Schnabel, D. Rueckert, and M.A. Viergever. Registration-Based Interpolation. IEEE Trans. Med. Imaging, 23(7):922-926, 2004. 